\title{
Equations of structural relaxation
}

\author{
M. Fuchs* and Th. VoigtmanN \\ Physik-Department, Technische Universität München, D-85747 Garching, \\ Germany
}

\begin{abstract}
In the mode coupling theory (MCT) of the liquid-glass transition the longtime structural relaxation follows from equations determined solely by equilibrium structural parameters. The present extension of these structural relaxation equations to arbitrarily short times on the one hand allows calculations unaffected by model assumptions about the microscopic dynamics and on the other hand supplies new starting points for analytical studies. As a first application, power-law like structural relaxation at a glass transition singularity is proved explicitly for a special schematic MCT model.
\end{abstract}

\section{$\S 1$. INTRODUCTION}

Dense liquids exhibit anomalously slow, temperature sensitive and non-exponentional relaxation processes, conventionally termed 'structural relaxation' because presumably cooperative rearrangements of particles are involved. Mode coupling theory (MCT) suggests that the evolution of the anomalous dynamics and its splitting off from the normal liquid dynamics is caused by bifurcation singularities in nonlinear retarded equations of motion for the (normalized) intermediate scattering functions $\Phi_{q}(t)=S_{q}(t) / S_{q}$ (Bengtzelius et al. 1984, Leutheusser 1984). These functions are some of the simplest specifying structural dynamics. The bifurcations of MCT, and the long-time dynamics in their vicinity, are determined solely by the equilibrium (static) structure factor $S_{q}$, (see Götze and Sjögren (1989) and Franosch et al. (1998)), and thus the MCT provides a frame to single out and to define structural relaxation. In this contribution the MCT equations for the structural relaxation are extended to arbitrarily short times, thus rendering them well defined even in the limit that the microscopic transient dynamics can be neglected. Whereas in Franosch et al. (1998) this was achieved by a discrete-dynamics model, here integral equations with explicit initial conditions are formulated. Thus the dependence on the initial conditions is displayed clearly. Additionally, monotonic solutions are guaranteed (Fuchs et al. 1991). Furthermore, for the structural dynamics equations obtained non-exponential relaxation can be proven for a special MCT model.

\section{§2. THE EQUATIONS OF MOTION OF MCT}

The MCT equations of motion for the autocorrelation functions of density fluctuations in simple liquids, $\Phi_{q}(t)$, are derived from Newton's equations with the 
Zwanzig-Mori formalism, leading to:

$$
\begin{gathered}
\partial_{t}^{2} \Phi_{q}(t)+\Omega_{q}^{2} \Phi_{q}(t)+\int_{0}^{t} \mathrm{~d} t^{\prime} M_{q}\left(t-t^{\prime}\right) \partial_{t^{\prime}} \Phi_{q}\left(t^{\prime}\right)=0 \\
\Phi_{q}(t \rightarrow 0)=1-\frac{1}{2}\left(\Omega_{q} t\right)^{2}
\end{gathered}
$$

and subsequent mode coupling approximations for the generalized longitudinal viscosity, finally resulting in

$$
\begin{aligned}
& M_{q}(t)=\Omega_{q}^{2} m_{q}(t)+M_{q}^{\mathrm{reg}}(t), \\
& m_{q}(t)=\sum_{\mathbf{k}+\mathbf{p}=\mathbf{q}} V(\mathbf{q} ; \mathbf{k}, \mathbf{p}) \Phi_{k}(t) \Phi_{p}(t) .
\end{aligned}
$$

In equation (1) the frequency $\Omega_{q}$ sets the microscopic timescale for the bare oscillators with wavevector $q$. In equation (3) viscous damping arises from mode-mode coupling, $m_{q}(t)$, equation (4), and from microscopic short-time dynamics denoted by $M^{\text {reg; }}$ see Götze (1991) for explicit expressions and discussions. A Laplace transformation of equations (1) and (3) using $\hat{\Phi}(s)=\int_{0}^{\infty} \mathrm{d} t \mathrm{e}^{-s t} \Phi(t)$ leads to

$$
\hat{\Phi}(s)=1 /\left[s+1 /\left[\hat{m}(s)+\left(s+\hat{M}^{\mathrm{reg}}(s)\right) / \Omega_{q}^{2}\right]\right] .
$$

The existence of a unique solution of the MCT equations which has all the properties of an autocorrelation function has been shown by Haussmann (1990). Further and more detailed properties could be proved by Götze and Sjögren (1995) upon the assumption of overdamped short-term motion. Let us mention two results relevant for this work, namely: (i) the existence of bifurcations in the equations derived from equations (1) and (4) for the long-time limits, $f_{q}=\lim _{t \rightarrow \infty} \Phi_{q}(t)$ (here the $f_{q}$ are the glass form factors and the bifurcations happen for critical values of the mode coupling vertices, $V=V^{\mathrm{c}}$, where $f_{q}=f_{q}^{\mathrm{c}}$ ); and (ii) for regular vertices, the $f_{q}$ depend smoothly on $V$, and there exists a longest relaxation time, $1 / \Gamma$, cutting off the final relaxation exponentially, $\Phi_{q}(t)-f_{q}=\mathcal{O}\left(e^{-\Gamma t}\right)$ for $V \neq V^{\mathrm{c}}$.

One of the simplest models exhibiting the generic features of the MCT bifurcation dynamics in equations (1)-(4) is obtained by the simplification of studying only one correlator, $\Phi(t)$, which experiences nonlinear feedback via one mode-coupling functional $m(t)=v_{1} \Phi(t)+v_{2} \Phi^{2}(t)$ with $v_{1,2} \geq 0$ (Götze 1984). We will start by discussing this so-called $F_{12}$ model before generalizing our results to the equations relevant for simple liquids.

\section{$\S 3$. ReSULTS FOR THE SCHEMATIC $F_{12}$ MODEL}

'Equations of structural relaxation' in the $F_{12}$ model are equations of motion determined solely by the vertices $v_{1}$ and $v_{2}$ which play the role of the static structural information entering the MCT vertices $V$ in equation (4). Bifurcations in the model lie on two lines parametrized by the exponent parameter $\lambda$ (Götze 1984): type-B lines, where $f^{\mathrm{c}}=1-\lambda>0$, at $v_{1}^{\mathrm{c}}=(2 \lambda-1) / \lambda^{2}$ and $v_{2}^{\mathrm{c}}=1 / \lambda^{2}$ with $1 / 2 \leq \lambda<1$, and type-A transitions, where $f^{\mathrm{c}}=0$, at $v_{1}^{\mathrm{c}}=1$ and $v_{2}^{\mathrm{c}}=\lambda$ for $0 \leq \lambda \leq 1$. Whereas in equations (1)-(4) the microscopic transient is required in order for solutions to exist (Götze 1991), after partial integration the short-time variation can be neglected, 


$$
\begin{aligned}
& \partial_{t}^{2} \Phi(t)+\int_{0}^{t} \mathrm{~d} t^{\prime} M^{\mathrm{reg}}\left(t-t^{\prime}\right) \partial_{t^{\prime}} \Phi\left(t^{\prime}\right) \ll \Omega^{2} \Phi(t), \text { leading to } \\
& \Phi(t)=m(t)-\frac{\mathrm{d}}{\mathrm{d} t} \int_{0}^{t} \mathrm{~d} t^{\prime} m\left(t-t^{\prime}\right) \Phi\left(t^{\prime}\right),
\end{aligned}
$$

while the expression for the memory function is not changed:

$$
m(t)=v_{1} \Phi(t)+v_{2} \Phi^{2}(t)
$$

Demanding the solutions to be of regular variation and that the Laplace transforms $\tilde{\Phi}(s)$ and $\hat{m}(s)$ exist, enabling one to use Tauber and Abel theorems (see e.g. Feller (1971)), the equations of structural relaxation have to be completed by specifying the initial variation

$$
\Phi(t) \rightarrow\left(t / t_{*}\right)^{-1 / 3} \quad \text { for } t \rightarrow 0 .
$$

In this case, the convolution integral, which will be abbreviated as $(m * \Phi)(t):=\int_{0}^{t} \mathrm{~d} t^{\prime} m\left(t-t^{\prime}\right) \Phi\left(t^{\prime}\right)$, leads to $(m * \Phi)(t \rightarrow 0) \rightarrow v_{2} t_{*} B(1 / 3,2 / 3)$, where $B(x, y)$ is Euler's beta function. The Laplace transform of equation (6) for all $s$ then is

$$
(1+s \hat{m}(s)) \hat{\Phi}(s)=\hat{m}(s)+v_{2} t_{*} B(1 / 3,2 / 3) .
$$

It is a straightforward exercise to verify that the last term in equation (9) is required for the existence of solutions with the required properties (Voigtmann 1998), thus leading to equation (8).

Note that the bifurcations to non-ergodic solutions cause $\hat{\Phi}$ and $\hat{m}$ to increase for small frequencies, suggesting neglecting $\left(s+\hat{M}^{\text {reg }}\right) \Omega^{-2} \ll \hat{m}(s)$ in equation (5) in order to derive equations for the structural relaxation alone. However, this procedure misses the non-analytical behaviour of $(m * \Phi)(t \rightarrow 0)$ connected to the limit $s \rightarrow \infty$.

One can extend equation (8) by a short-time series expansion (see appendix A):

$$
\Phi(t)=\left(t / t_{*}\right)^{-1 / 3}\left(1+\sum_{n=1} c_{n}\left(t / t_{*}\right)^{n / 3}\right) \quad t<r t_{*} .
$$

Figure 1 shows the numerically obtained radii of convergence $r$ of equation (10) along the line of bifurcations in the $F_{12}$ model.

Figure 2 shows numerical solutions of equations (6)-(8) which exhibit slow relaxation processes upon approach of a bifurcation singularity at critical values of the coupling vertices. This explains the sensitive dependence of the dynamics on small regular changes in $\varepsilon$, which measures the distance to the singularity. The algorithm described in Fuchs et al. (1991) is well suited to numerical integration. A type-B fold bifurcation at $\varepsilon=0$ separates ergodic, $\Phi(t \rightarrow \infty)=f=0$ for $\varepsilon<0$, from non-ergodic dynamics, $f \geq f^{c}>0$ for $\varepsilon \geq 0$. Asymptotic expansions identify two divergent timescales connected with two power law relaxation windows (Götze 1984). As demonstrated, e.g. in Franosch et al. (1998), the long-time solutions of the complete MCT equations of motion, equations (1)-(4), become independent of the microscopic transient. Over long times they agree with the solutions of the equations of structural relaxation, equations (6) and (7). This is exemplified in figures 2 and 3 using two approximations for the regular microscopics in the $F_{12}$ model: undamped oscillation, $M^{\text {reg }} \equiv 0$, and pure relaxation, $\left(s+\hat{M}^{\mathrm{reg}}(s)\right) \Omega^{-2} \rightarrow \Gamma_{0}$. It is apparent 


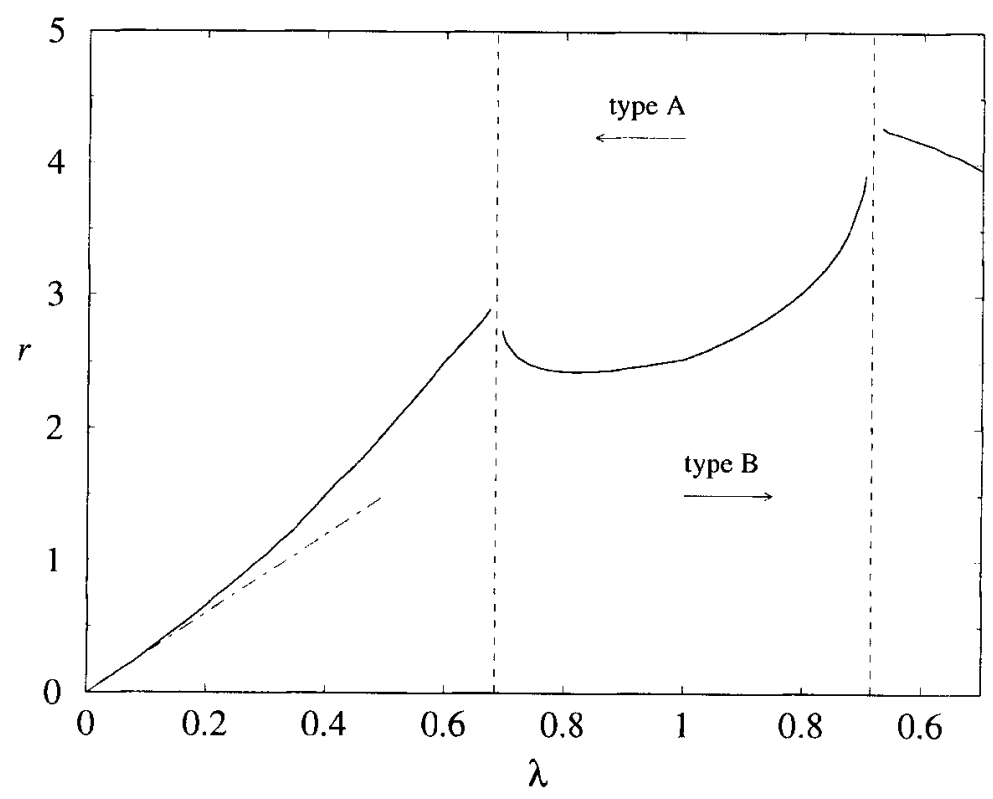

Figure 1. Radius of convergence $r$ of equation (10) numerically obtained using the CauchyHadamard criterion and equation (A 2) along the line of bifurcations in the $F_{12}$ model. The line parametrized by $\lambda$ is given by $v_{2}^{c}=1 / \lambda^{2}$ and $v_{1}^{c}=(2 \lambda-1) / \lambda^{2}$ for type-B $\left(f^{\mathrm{c}}>0\right)$, and by $v_{2}^{\mathrm{c}}=\lambda$ and $v_{1}^{\mathrm{c}}=1$ for type-A $\left(f^{\mathrm{c}}=0\right)$ transitions. The vertical dashed lines indicate $\lambda_{*}$ where equation (10) with $c_{n>1} \equiv 0$ holds for all times; i.e., $r \rightarrow \infty$. The dot-dashed line describes the linear decrease, $r \propto \lambda$, see appendix A, upon approaching the $F_{1}$ model at $\left(v_{1}^{\mathrm{c}}, v_{2}^{\mathrm{c}}\right)=(1,0)$.

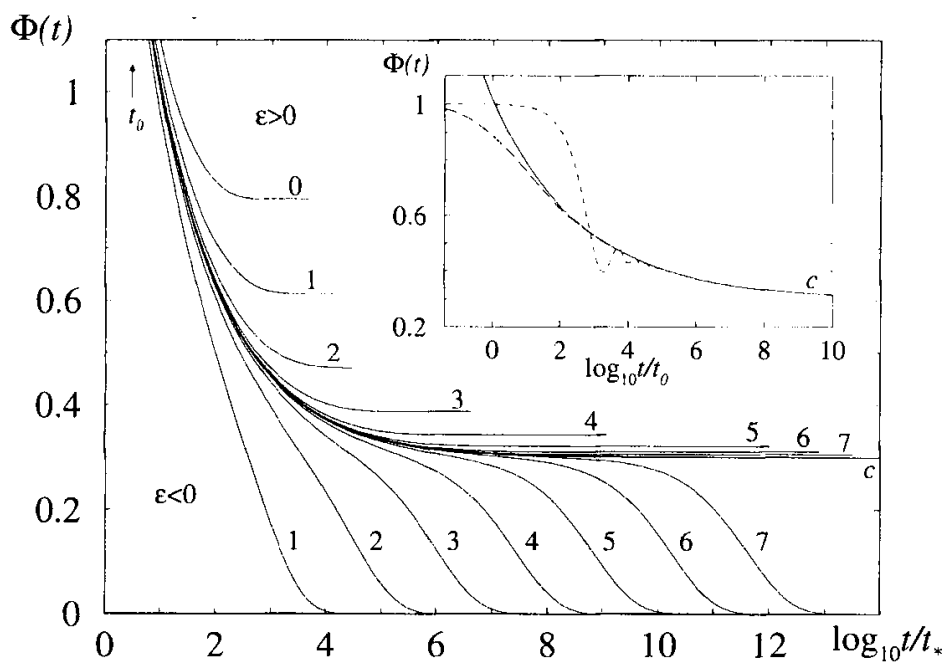

Figure 2. Correlators $\Phi(t)$ obtained from equations (6) and (7) for exponent parameter $\lambda=0.70$. The bold line marked by a $c$ corresponds to the bifurcation point and the others are calculated for $v_{1,2}=v_{1,2}^{\varepsilon}(1+\varepsilon), \varepsilon= \pm 1 / 4^{n}, n=0,1, \ldots$ as labelled. The timescale $t_{0}$ from equation (11) is indicated and used in the inset to compare the curve at $v^{c}$ to one crossing over to oscillations at short times (dashed line calculated for the $F_{12}$ model from equations (1)-(4) with $M^{\text {reg }} \equiv 0$ ) and to another with overdamped short-term dynamics $\left(\left(s+\hat{M}^{\mathrm{reg}}(s)\right) \Omega^{-2} \rightarrow \Gamma_{0}\right.$, long dashes). 


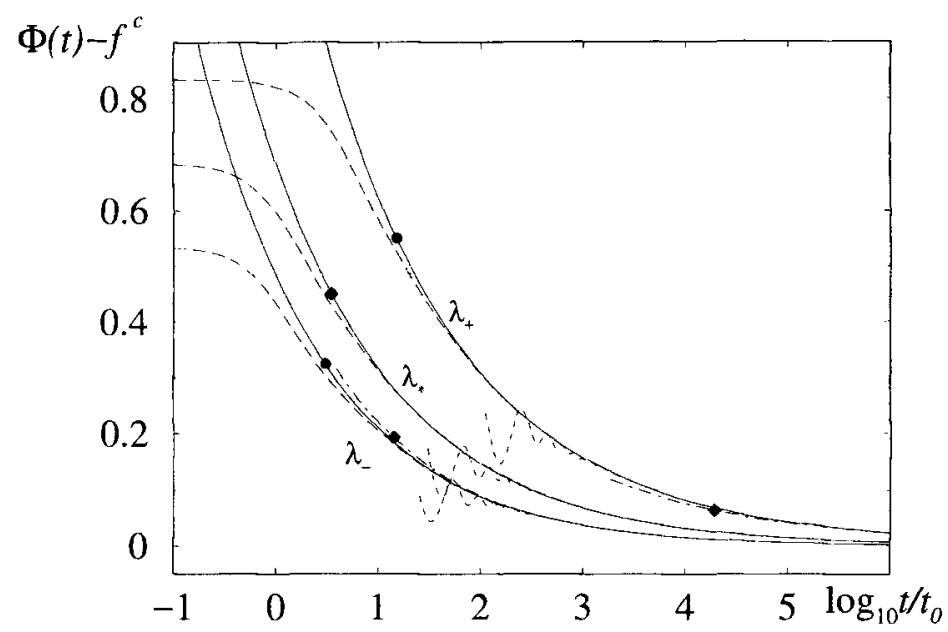

Figure 3. Correlators at bifurcation points of the $F_{12}$ model for different microscopic transients and shifted using $f^{\mathrm{c}}$ are plotted versus rescaled time, with $t_{0}$ from equation (11). Solutions for exponent parameters $\lambda_{*}=0.684$ and $\lambda_{ \pm}=\lambda_{*} \pm 0.15$ as labelled are shown obtained from the equations of structural relaxation (equations (6) and (7), solid lines), and from equations (1)-(4) with undamped oscillatory $\left(M^{\text {reg }} \equiv 0\right.$, short dashed lines terminated for $\left.t \lesssim 10^{2} t_{0}\right)$ and with overdamped $\left(\left(s+\hat{M}^{\text {reg }}(s)\right) \Omega^{-2} \rightarrow \Gamma_{0}\right.$, long dashes) short-time dynamics. Dash-dotted curves indicate the critical decay law $\left(t_{0} / t\right)^{a}$ with $a_{+}=0.26$ and $a_{-}=0.39$ corresponding to the $\lambda_{ \pm}$. For $\lambda_{*}$, where $a_{*}=1 / 3$, the structural relaxation follows the critical decay for all times. Black circles (diamonds) indicate where the structural dynamics (critical decay) curves deviate by $20 \%$ in horizontal direction from the solutions with overdamped microscopics.

that oscillatory short-time dynamics mask the structural relaxation more strongly than relaxational.

Assuming regular variation of the solutions of equations (1)-(4), at a bifurcation point, power law relaxation could be argued:

$$
\Phi(t)=f^{\mathrm{c}}+h\left(t / t_{0}\right)^{-a}, \quad \text { for } t \rightarrow \infty \quad \text { and } v_{1,2}=v_{1,2}^{\mathrm{c}},
$$

where the exponent $a$ follows from the exponent parameter $\lambda$, which is a function of $v_{1,2}^{\mathrm{c}}$, via $\lambda=B(1-a, 1-a)(1-2 a)$ (Götze 1984). From the equations of structural relaxation, equations (6) and (7) and the short-time expansion (equation (10)), one concludes that this asymptote requires $t>t_{*} r$ in general. The transient time $t_{0}$ is obtained from matching equation (11) to equation (10), while the critical amplitude $h=\left(1-f^{\mathrm{c}}\right)$ for the $F_{12}$ model.

In the $F_{12}$ model there exist two points where the short-time expansion can be found explicitly and solves the equations of structural relaxation, equations (6)-(8), for all times, i.e., $r \rightarrow \infty$ in equation (10) (see appendix $\mathrm{A}$ and figure 1):

$$
\Phi(t)=f^{\mathrm{c}}+\left(t / t_{*}\right)^{-1 / 3} \quad \text { for } \quad \lambda=\lambda_{*}=\frac{1}{3} B\left(\frac{2}{3}, \frac{2}{3}\right)=0.684 \ldots \quad \text { and } v_{1,2}=v_{1,2}^{\mathrm{c}} \text {. }
$$

Both points are bifurcation points, as finite distances from the singularities lead to the existence of long-time relaxation rates as shown in appendix $B$ (see also (Götze (1984, 1991)). Furthermore, the initial condition, equation (8), requires for the critical exponent $a=a_{*}=1 / 3$, which is satisfied for $\lambda=\lambda_{*}$. Thus, equation (12) verifies power-law relaxation at a bifurcation singularity of equations describing the structural relaxation of a schematic MCT model. 
The existence of a solution of the integral equations for all times at a singular point also allows for these special cases to deduce a long-time solution for small distances from the bifurcation in an expansion which can be expected to have a finite radius of convergence, $\left(t / t_{0}\right)|\varepsilon|^{3 / 2}<r_{\beta}$.

$$
\Phi(t) \rightarrow\left(t / t_{0}\right)^{-1 / 3}\left[1+\sum_{n=1} A_{n}\left(\varepsilon\left(t / t_{0}\right)^{2 / 3}\right)^{n}\right] \quad \text { for } t \rightarrow \infty .
$$

Therefore the calculation sketched in appendix $B$ introduces the $\beta$ timescale, $t_{\varepsilon}=t_{0}|\varepsilon|^{-1 / 2 a}$ as $a=1 / 3$ for $\lambda=\lambda_{*}$, and agrees with the expansion of the so-called $\beta$ correlator for small rescaled times $\tilde{t}=t / t_{\varepsilon}$; (see Götze (1984)). There it is shown also, for general $\lambda$, how to extend this solution to even longer times. Note that the expansion carried through in appendix $B$ does not require a matching to the critical decay.

\section{$\S 4$. EXTENSIONS TO MCT EQUATIONS FOR SIMPLE LIQUIDS}

The extension of the analysis of the bifurcation dynamics of schematic models to the full MCT equations for simple liquids, equations (1)-(4), has been achieved in Götze (1985) and rests upon the central-manifold concept. The generalization of the equations of structural relaxation to coupled, wavevector dependent equations is trivial; equation (6) attains indices $q$ and equation (7) is replaced by equation (4). For quadratic polynomials in equation (4), the power law of the initial condition is not changed:

$$
\Phi_{q}(t) \rightarrow x_{q} t^{-1 / 3} \quad \text { for } t \rightarrow 0 ; x_{q} \geq 0
$$

It is surprising that a vector $x_{q}$ has to be specified in order to render the solutions unique; recall that owing to the factorization property of the critical decay, $\Phi_{q}(t)=f_{q}^{c}+h_{q}\left(t / t_{0}\right)^{-a}$ (Götze 1985), the long-time dynamics of the structural relaxation become unique by specifying only the one matching time $t_{0}$.

From simple generalizations of the calculations presented in appendix $\mathrm{A}$ it is obvious that the short-time expansion, equation (10), can be obtained in the wavevector dependent case also.

\section{§5. Conclusions}

Definitions of equations for structural relaxation not restricted to long times are given within the MCT. These equations (equations (4) and (6)) together with the initial conditions (equation (14)) determine smooth but sensitively varying intermediate scattering functions $\Phi_{q}(t)$ from the equilibrium structure of the liquid; the static structure factor $S_{q}$ is the only quantity appearing in the equations of motion. The possibility of specifying an arbitrary initial non-negative amplitude $x_{q}$ is worth stressing, and holds also for the discrete dynamics model of Franosch et al. (1998). The short-time divergence of the solutions of the structural relaxation equations renders them unphysical for particularly short times; they violate the imposed normalization of $\Phi_{q}(t)$. This stresses that the physical mechanism (the so-called 'cage effect') causing structural relaxation is relevant for long-time dynamics only. Any complete description of the density fluctuations requires a transient to regular microscopic short-time dynamics. Nevertheless, extending the structural relaxation equations to all times on the one hand allows the definition of a lower limit of validity of this long-time description (the time where the solutions increase above unity could be 
chosen) and on the other hand presents a conceptual simplification as some transient effects are eliminated. Thus for example, analytical proofs of power-law relaxation at bifurcation points become possible for special cases; recall that at regular points of the full MCT equations a longest relaxation time exists. Also, the smaller of the two divergent timescales of MCT could be identified from a perturbation expansion. Moreover, the aspect that MCT equations of motion with different models for short-time microscopic motion lead to identical long-time dynamics again is observed.

\section{ACKNOWLEDGEMENTS}

We thank Professor W. Götze for discussions and critical reading of the manuscript. The work was supported by the Deutsche Forschungsgemeinschaft under Contract Fu 309/2-1 and by Verbundprojekt BMBF 03G05TUM.

Appendix A

Entering the ansatz $\Phi(t)=f^{\mathfrak{c}}+\phi(t)$ into equations (6) and (7) leads to

$$
\check{\sigma}=\bar{\sigma} \phi(t)-\bar{\lambda} \phi^{2}(t)+\left(1 / \lambda^{\prime}+\tilde{\sigma}\right)(\phi \dot{*} \phi)(t)+\left(\phi^{2} \dot{*} \phi\right)(t),
$$

where a dot denotes a time derivative and the following abbreviations are used: $\bar{\lambda}=\lambda / \lambda^{\prime}, \quad \lambda^{\prime}=\lambda /\left(1-f^{\mathfrak{c}}\right), \quad \check{\sigma}=\left(1-f^{\mathfrak{c}}\right)^{2} \sigma / \Delta, \quad \sigma=\left(1-f^{\mathfrak{c}}\right)\left(\delta v_{1} f^{\mathfrak{c}}+\delta v_{2} f^{\mathrm{c}}\right)$, $\Delta=\left(\lambda^{\prime}+\left(1-f^{\mathfrak{c}}\right)^{2} \delta v_{2}\right), \quad 1 / \lambda^{\prime}+\tilde{\sigma}=\left[1+\left(1-f^{c}\right)^{2}\left(\delta v_{1}+2 \delta v_{2} f^{c}\right)\right] / \Delta, \quad$ and $\bar{\sigma}=\left(1-f^{c}\right)(1+\tilde{\sigma}-(1+\sigma) / \Delta)$ which are obtained from $v_{1,2}=v_{1,2}^{\mathrm{c}}+\delta v_{1,2}$, where the limit of $v_{2}$ approaching zero has been excluded. In the vicinity of a type-B transition, $\lambda^{\prime}=1, \bar{\lambda}=\lambda$. Close to a type-A transition $\lambda^{\prime}=\lambda$, and thus $\bar{\lambda}=1$. From the short-time expansion (equation (10)), one obtains a recursion relation for $\tilde{c}_{n}$, where $\tilde{c}_{0}=1, \tilde{c}_{1}=c_{1}-f^{c}$ and $\tilde{c}_{n}=c_{n}$ else, and $\delta_{n, m}$ is a Kronecker symbol:

$$
\begin{aligned}
\tilde{c}_{n+1}= & \frac{3 /(n+1)}{\left(2 B\left(\frac{n+2}{3}, \frac{2}{3}\right)+B\left(\frac{n+3}{3}, \frac{1}{3}\right)\right)} \\
& \times\left\{\sum_{m=0}^{n}\left[\bar{\lambda}-\frac{n+1}{3} B\left(\frac{n-m+2}{3}, \frac{m+2}{3}\right)\left(1 / \lambda^{\prime}+\tilde{\sigma}\right)\right]\right. \\
& \times \tilde{c}_{n-m} \tilde{c}_{m}+\check{\sigma} \delta_{n, 2} \\
& -\left(1-\delta_{n, 0}\right)\left[\bar{\sigma} \tilde{c}_{n-1}+\frac{n+1}{3} \sum_{m=1}^{n}\left(\sum_{k=0}^{m} B\left(\frac{n-k+2}{3}, \frac{k+2}{3}\right)\right.\right. \\
& \left.\left.\left.\times \tilde{c}_{m-k} \tilde{c}_{k}+B\left(\frac{n+2}{3}, \frac{2}{3}\right) \tilde{c}_{m}\right) \tilde{c}_{n+1-m}\right]\right\} .
\end{aligned}
$$

At two singular points, where $\check{\sigma}=\bar{\sigma}=\tilde{\sigma}=0$ and $\lambda=\lambda_{*}=B\left(\frac{2}{3}, \frac{2}{3}\right) / 3=0.684 \ldots$, all $\tilde{c}_{n}$ vanish. This proves equation (12). Approaching the $F_{1}$ model at $\left(v_{1}, v_{2}\right)=\left(v_{1}, 0\right)$ (Götze 1991), the radius of convergence of equation (10) shrinks since for an $m$ th order polynomial mode coupling functional a similar expansion with $\Phi(t) \rightarrow\left(t / t_{*}\right)^{-1 /(m+1)}$ for $t \rightarrow 0$ applies (Voigtmann 1998). 


\section{APPENDIX B}

Close to the type-B transition at $\lambda=\lambda_{*}$, equation (A 1) can be expanded around the solution $\phi_{0}(t)=t^{-1 / 3}$ at $\delta v_{1,2}=0$. Using $\phi(t)=\phi_{0}(t)+\phi_{1}(t)$, specifying the path such that $\tilde{\sigma}=0$ for simplicity and integrating this leads to

$$
\int_{0}^{t} \mathrm{~d} s K(t, s) \phi_{1}(s)=\check{\sigma} t-\frac{2}{3} \bar{\sigma} t^{2 / 3}-\left(\left(\bar{\sigma}+\phi_{1}+2 \phi_{0} \phi_{1}\right) * \phi_{1}\right)(t)+\left(\left(\lambda_{*}-\phi_{0}-\phi_{1}\right)_{*} \phi_{1}^{2}\right)(t)
$$

where the Volterra kernel is of generalized Abel form:

$$
K(t, s)=2(t-s)^{-1 / 3}-2 \lambda_{*} s^{-1 / 3}+(t-s)^{-2 / 3}+2(t-s)^{-1 / 3} s^{-1 / 3} .
$$

From the short-time expansion (equation (10)), $\phi_{1}(t \rightarrow 0) \propto t^{1 / 3}$ can be deduced in this case, ruling out that kernel $K$ has eigenvalue zero. The expansion $\phi_{1}(t)=\sum_{n=1} \sigma^{n} g^{(n)}(t)$ thus leads to a recursive set of solvable linear integral equations for the $g^{(n)}$. If their long-time behaviour is sought, only the first two terms in $K$ need to be considered, and by induction one shows that $g^{(n)}(t) \rightarrow A_{n} t^{(2 n-1) / 3}$ for $t \rightarrow \infty$, where the $A_{n}$ obey $A_{1}=1 /\left(2\left(B\left(\frac{2}{3}, \frac{4}{3}\right)-\lambda_{*}\right)\right)$ and

$$
\begin{aligned}
A_{n}= & -\frac{1}{2}\left[\sum_{m=1}^{n-1} A_{n-m} A_{m}\left(B\left(\frac{2(n-m+1)}{3}, \frac{2 m+2}{3}\right)-\lambda_{*} \frac{2 n+1}{3}\right)\right] /\left(B\left(\frac{2}{3}, \frac{2 n+2}{3}\right)\right. \\
& \left.-\lambda_{*} \frac{2 n+1}{3}\right) .
\end{aligned}
$$

Replacing $\sigma$ with $\varepsilon$, this coincides with equation (13) and the short-time expansion of the $\beta$-correlator for $\lambda=\lambda_{*}$ (Götze 1984).

\section{REFERENCES}

Bengtzelius, U., Götze, W., and Suölander, A., 1984, J. Phys. C., 17, 5915.

FELLER, W., 1971, An Introduction to Probability Theory and its Applications, Vol. 2 (New York! Wiley).

Franosch, T., Götze, W., MaYr, M. R., and Singh, A. P., 1998, J. non-crystalline Solids, 235-237, 66.

Fuchs, M., Götze, W., Hofacker, I., and Latz, A., 1991, J. Phys.: condens. Matter, 3, 5047.

Götze, W., 1984, Z. Phys. B, 56, 139; 1985, Z. Phys. B, 60, 195; 1991, Liquids Freezing and Glass Transition, edited by J. P. Hansen, D. Levesque and J. Zinn-Justin (Amsterdam. North Holland), p. 287.

Götze, W., and Sjögren, L., 1989, J. Phys.: condens. Matter, 1 4183; 1995, J. Math. Anal. Appl., 195, 230.

Haussmann, R., 1990, Z. Phys. B, 79, 143.

Leutheusser, E., 1984, Phys. Rev. A, 29, 2765.

VolgtmanN, TH., 1998, Diploma thesis, Technische Universität München. 